\title{
Muscles Transfer around the Shoulder in Cases of Brachial Plexus Birth Palsy
}

\author{
Hesham El Sobkey \\ Department of Neurosurgery, Mansoura University, Mansoura, Egypt \\ Email: h_espero@yahoo.com
}

How to cite this paper: El Sobkey, $\mathrm{H}$. (2019) Muscles Transfer around the Shoulder in Cases of Brachial Plexus Birth Palsy. Open Journal of Modern Neurosurgery, 9, 269-280. https://doi.org/10.4236/ojmn.2019.93026

Received: May 22, 2019

Accepted: June 21, 2019

Published: June 24, 2019

Copyright (C) 2019 by author(s) and Scientific Research Publishing Inc. This work is licensed under the Creative Commons Attribution International License (CC BY 4.0).

http://creativecommons.org/licenses/by/4.0/

\begin{abstract}
Background: Disabling internal rotation contractures are frequently experienced in children with unresolved birth brachial plexus palsies. Multiple surgical options like muscle release, tendon transfer, or humeral osteotomy are available to treat such cases. Purpose: Evaluation of the outcome of subscapularis release and latissimus dorsi and teres major tendon transfer in the management of obstetric brachial palsies in Mansoura University neurosurgical department. Study type: Retrospective observational study. Patients and Methods. Twenty-five cases who underwent subscapularis release and latissimus dorsi and teres major transfer were included in the study. All patients were subjected to complete history taking, through clinical examination. The degree of shoulder movement and disability was assessed via Modified Gilbert shoulder evaluation scale. Results. The least follow up period for our patients was 9 months. There was a clear improvement of shoulder function evaluated using Modified Gilbert shoulder evaluation scale as there were 73\% of postoperative group between GIV and GV while about $84 \%$ of preoperative group were between GII and GIII. Conclusion: Tendon transfer is a valid easy procedure for correction of shoulder deformities in patients with obstetrical brachial plexus palsy. It is considered a very good option for patients who missed the chance of microsurgical repair or patients with poor shoulder recovery after surgery. Although some authors reported deterioration of shoulder function with log time follow up after tendon transfer, it is still better than those who were not operated.
\end{abstract}

\section{Keywords}

Brachial Plexus, Tendon Transfer, Shoulder

\section{Introduction}

Neonatal brachial plexus palsies (NBPP) are a clinical entity that is identified 
immediately after birth as a result from trauma to the fetal brachial plexus that may occur before completion of delivery. The exact timing of that pathology is still not completely understood. Although the majority of researchers believe that it occurs during the second stage of labour during shoulder extraction, other assume that it may occur before delivery as a consequence of endogenous or exogenous force to which the fetus is exposed [1].

NBPP have been reported to affect 1.5 per 1000 live birth, and that ratio has not been decreased in recent days [2]. The majority of these injuries are transient in nature and function will be restored completely in about $70 \%-92 \%$ of cases, while in other cases, permanent disabling functional outcomes are also reported [3].

NBPP have six clinical subtypes including: complete (total) brachial plexus palsy, Duchenne-Erb palsy, upper-middle trunk brachial palsy, Klumpke palsy, fascicular palsy, and bilateral brachial plexus palsy [4]. Of note, Duchenne-Erb and Klumpke palsies are the most commonly encountered types in the obstetric practice [1].

The etiopathogenesis of such condition can be attributed to compression, traction, vascular disruption, or inflammation, and according to the degree of injury, permanent or transient neurological deficits may result. Intrauterine compression can occur in cases with uterine anomalies, masses, or pelvic masses during pregnancy, and such cases usually have an obvious deformity in the upper limb at the time of delivery [5] [6].

Because of muscle weakness and soft tissue contractures, functional limitations may arise in children who experienced incomplete recovery from such injury. Both bone growth and joint development are affected by this muscular imbalance that may result in glenohumeral deformity on the long-term [7] [8].

Although there is a global agreement that the disturbance in muscle power and contraction should be corrected, there is no consensus on how to perform that correction. Release of internal shoulder rotators along with contracted tissues, transfer of tendons that augment abduction and external rotation are available options. Some surgeons prefer to combine both procedures [9].

Rotational osteotomy is recommended for older patients who present with advanced deformity of the glenohumeral joint. In addition, latissimus dorsi and teres major tendon transfer are the commonest transfers to be done for such cases to restore external rotation and abduction. However, there is no clear evidence that supports the superiority of one technique over the other [10].

Subscapularis contracture may hinder the functional rotators to act normally, as it is considered the largest rotator cuff muscle and thus, subscapularis release is of crucial importance to restore the limited shoulder movements. This contracture is assessed clinically by a decrease in shoulder passive external rotation with the arm along the trunk and the elbow in $90^{\circ}$ flexion [11].

Therefore, we can notice the clear agreement that shoulder deformity and limited mobility is a common complication of obstetrical brachial plexus palsy. 
This deformity affects patient daily activity and adds more burden to his suffering. On the other hand, there are different surgical options, multiple techniques and absence of solid consensus about the management strategies of these deformities and timing of surgeries. We conducted this study to evaluate the outcome of subscapularis release, latissimus dorsi and teres major tendon transfer in the management of obstetric brachial nerve palsies.

\section{Patient and methods}

\subsection{Study Design}

This is a retrospective study of patients presented with shoulder deformity and limited mobility due to obstetrical brachial plexus birth injury who presented at Mansoura University Hospitals neurosurgical clinics from 2014 to 2017. The study was approved and accepted by the local ethical committee.

\subsection{Patient Sample}

Twenty-five patients $(\mathrm{n}=25)$ including 11 males and 14 females were included in our study (Table 1).

\subsection{Inclusion Criteria}

1) Obstetrical brachial plexus palsy.

2) Adequate power of latissimus dorsi and terse major muscles.

3) Possible shoulder abduction and external rotation.

\subsection{Exclusion Criteria}

1) Sever shoulder contracture with difficult passive exercise.

2) Patients older than $18 y$ old.

3) Flail limb with weak hand grip.

\subsection{Patient Consent}

A written formal consent was obtained from patients after the explanation of the details, advantages and drawbacks of the surgical procedure.

\subsection{Patient Evaluation}

Thorough medical history was taken from all patients with careful reviewing of both labor and delivery history. We also reviewed all relevant events throughout the patient's life including their limbs power improvement, all medications they were commenced on, physiotherapeutic programs and any previous surgical procedures.

A modified Gilbert shoulder evaluation scale was used to assess the shoulders of these children as a preoperative evaluation and in regular follow up to evaluate shoulder improvement as shown in Table 2. All these children should have possible passive arm abduction and external rotation, enough muscle strength of the latissimus dorsi and adequate elbow and hand function. 
Table 1. Demographic data of the patients.

\begin{tabular}{|c|c|c|c|c|c|}
\hline & Age in months & Sex & Side & Delivery & Previous Surgery \\
\hline 1 & 24 & Female & Right & CS & $\mathrm{NO}$ \\
\hline 2 & 27 & Female & Right & CS & NO \\
\hline 3 & 626 & Female & Right & CS & NO \\
\hline 4 & 32 & Male & Right & CS & YES \\
\hline 5 & 48 & Female & Left & CS & YES \\
\hline 6 & 123 & Male & Right & CS & NO \\
\hline 7 & 144 & Female & Right & CS & NO \\
\hline 8 & 34 & Male & Right & CS & $\mathrm{NO}$ \\
\hline 9 & 47 & Female & Right & CS & YES \\
\hline 10 & 52 & Male & Right & CS & NO \\
\hline 11 & 31 & Female & Right & CS & YES \\
\hline 12 & 24 & Male & Right & CS & NO \\
\hline 13 & 27 & Male & Right & CS & NO \\
\hline 14 & 25 & Female & Left & CS & NO \\
\hline 15 & 29 & Male & Right & CS & NO \\
\hline 16 & 46 & Male & Right & CS & NO \\
\hline 17 & 34 & Female & Right & CS & NO \\
\hline 18 & 50 & Female & Right & CS & YES \\
\hline 19 & 24 & Male & Left & CS & NO \\
\hline 20 & 32 & Male & Right & CS & $\mathrm{NO}$ \\
\hline 21 & 26 & Male & Right & CS & NO \\
\hline 22 & 27 & Female & Right & CS & $\mathrm{NO}$ \\
\hline 23 & 48 & Female & Right & CS & YES \\
\hline 24 & 24 & Female & Right & CS & NO \\
\hline 25 & 27 & Female & Right & CS & YES \\
\hline
\end{tabular}

Table 2. Modified Gilbert shoulder evaluation scale [12].

\begin{tabular}{cc}
\hline Grade & Joint description \\
\hline 0 & Flail shoulder. \\
I & Abduction $=45^{\circ}$, no active external rotation. \\
II & Abduction $<90^{\circ}$, no active external rotation. \\
III & Abduction $=90^{\circ}$, active external rotation $<30^{\circ}$. \\
IV & Abduction $<120^{\circ}$, active external rotation $10^{\circ}$ to $30^{\circ}$. \\
V & Abduction $>120^{\circ}$, active external rotation $30^{\circ}$ to $60^{\circ}$. \\
VI & Normal shoulder. \\
\hline
\end{tabular}




\subsection{Preoperative Preparation}

An extensive physiotherapeutic program was applied to these children as a preoperative preparation to improve shoulder functions and increase the range of passive movement. Patients were prepared for surgery after routine labs and preoperative medical evaluation.

\subsection{Surgical Procedure}

Surgery was done under general anesthesia. Infants were put in lateral position on the operating table with the affected arm draped free and able to move in all directions. The surgical incision was infiltrated with 1:100,000 concentration of saline adrenaline for a bloodless operative field. The first incision as in Figure 1 was taken at the posterior axillary fold going across the axillary skin fold to expose and detach the latissimus dorsi and teres major muscle tendons from their humeral insertion.

These tendons were then elevated up to their neurovascular pedicle that was found proximally along the lateral scapular border. A great care was taken not to disrupt this neurovascular bundle for the success of our repair.

Another small incision was made at the upper surface of the shoulder at the planned site for insertion. Both tendons were anchored by $2 \backslash 0$ nylon suture and then mobilized through a tunnel under the dorsal part of the deltoid muscle to be reinserted in the tendon of the rotator cuff at the greater tuberosity of the humerus as shown in Figure 2.

Then a silk suture was applied to the lower tip of the scapula to help in its elevation and fixation during subscapularis release. A dissector was introduced through the undersurface of the lateral border of the scapula gently separating it from the subscapularis muscle then a drain was inserted in this space and anatomical closure was accomplished.

\subsection{Postoperative Care}

The shoulder was immobilized in 120-degree abduction and 90-degree external rotation in a plaster cast for 6 weeks. After cast removal, we started a condensed physiotherapeutic program for at least six months.

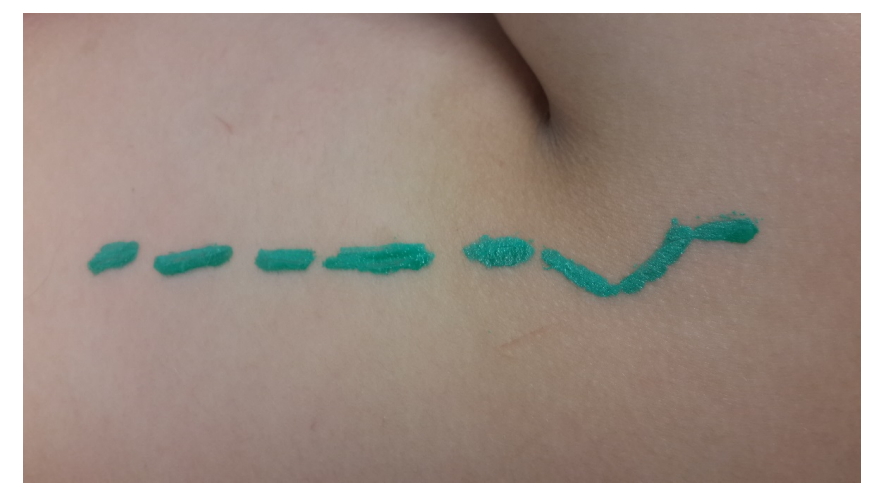

Figure 1 . The $1^{\text {st }}$ skin incision at the posterior axillary fold. 


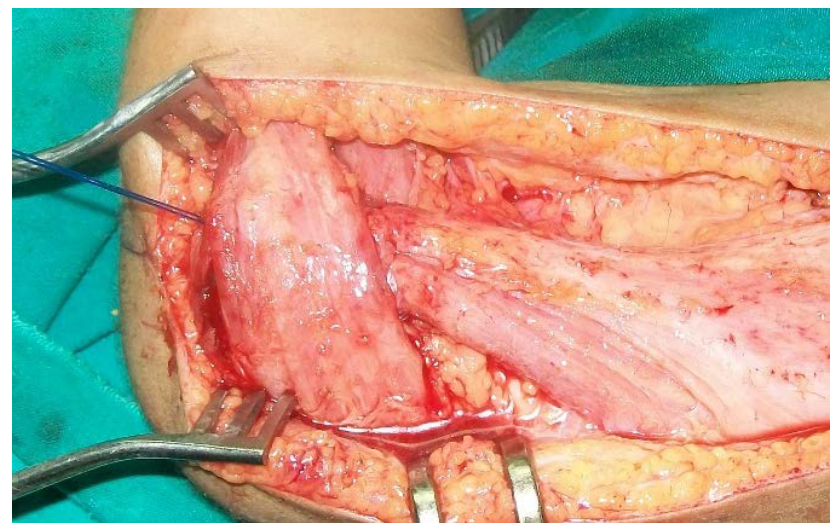

Figure 2. Tunneling of the tendons of latissimus dorsi and terse major muscles undersurface of the deltoid muscle.

\subsection{Follow Up}

We used Modified Gilbert shoulder evaluation scale to evaluate the shoulder recovery of our patients. We evaluate our patients immediately after cast removal and then every three months for at least nine months some of our patients were followed up for four years. The amazing finding is that some patients showed shoulder improvement immediately after cast removal.

\subsection{Statistical Analysis}

The collected data were coded, processed and analyzed using the SPSS (Statistical Package for Social Sciences) version 22 for Windows ${ }^{\circledast}$ (SPSS Inc, Chicago, IL, USA). Qualitative data was presented as number (frequency) and Percent. Quantitative data was tested for normality by Kolmogorov-Smirnov test and was expressed as median (range). Chi square test $\left(\mathrm{X}^{2}\right)$ was used to compare two groups with categorical variables. $P$ value $\leq 0.05$ was considered significant.

\section{Results}

We included 25 patients; eleven of them (44\%) were males and the remaining 14 cases (66\%) were females. These cases had a mean age of 40.6 months (range, 2 12 years). Of note, 15 cases (60\%) had an age below 3 years while two of them (8\%) were above 10 years old. Right arm was affected in twenty-two patients $(88 \%)$. All these cases $(100 \%)$ were delivered via the vaginal route; moreover, nineteen of them $(76 \%)$ were the $1^{\text {st }}$ child to their mothers (Table 3 ).

Eighteen patients $(72 \%)$ were not previously operated while seven patients (28\%) underwent previous microsurgical exploration and repair with limited shoulder improvement. Seventeen patients $(68 \%)$ had a total palsy whereas eight patients (32\%) had an upper palsy. All our patients showed improvement of their shoulder power and range of movement.

Table 4 shows a comparison between preoperative and postoperative shoulder function according to Modified Gilbert shoulder evaluation scale in these children. The majority of children the preoperative group sixteen (64\%) was of GII and GI while in postoperative group eighteen (72\%) was of GIV and GV. 
Table 3. Demographic and clinical data.

\begin{tabular}{cc}
\hline Variable & Data \\
\hline Age (years) & $3.38(2-12)$ \\
Gender & $11(44 \%)$ \\
-Male & $14(56 \%)$ \\
-Female & \\
Affected side & $22(88 \%)$ \\
-Right & $3(12 \%)$ \\
-Left & \\
Mode of delivery & $25(100 \%)$ \\
-Vaginal & $0(0 \%)$ \\
-Caesarian section & $7(28 \%)$ \\
Previous microsurgery & \\
Type of palsy & $8(32 \%)$ \\
-Upper trunk & $17(68 \%)$ \\
-Total palsy & $3(12 \%)$ \\
Blood transfusion & $1(4 \%)$ \\
Surgical site infection & $2(8 \%)$ \\
Pressure scores & \\
\hline
\end{tabular}

Table 4. Comparing preoperative and postoperative Gilbert shoulder scale.

\begin{tabular}{cccc}
\hline Grade & Preoperatively & Postoperatively & P value \\
\hline 0 & $0(0 \%)$ & $0(0 \%)$ & \\
I & $4(16 \%)$ & $0(0 \%)$ & \\
II & $12(48 \%)$ & $1(4 \%)$ & $\mathrm{P}<0.001$ \\
III & $9(36 \%)$ & $6(24 \%)$ & \\
IV & $0(0 \%)$ & $13(52 \%)$ & \\
V & $0(0 \%)$ & $5(20 \%)$ & \\
VI & $0(0 \%)$ & $0(0 \%)$ & \\
\hline
\end{tabular}

As illustrated in Table 4 there is clear improvement of shoulder function evaluated using Modified Gilbert shoulder evaluation scale as there were $73 \%$ of postoperative group between GIV and GV while about $84 \%$ of preoperative group were between GII and GIII.

\subsection{Complications}

Three of our patients (12\%) required blood transfusion, and all of them were less than three years old. One patient (4\%) had superficial surgical site infection which improved by medical treatment and dressing. One patient had a stich sinus at the shoulder which was treated with antibiotics with recurrent attacks of infection. After six weeks, the cast was removed, the wound was explored, and the prolene stitches were removed. The tendons were firmly healed and the patient was allowed to continue his physiotherapeutic program after that. 
Another girl, who was known to have Attention Deficit Hyperactivity Disorder (ADHD), broke the plaster cast and thus, we instructed the parents to fix the cast with a Crip bandage and they came to our facility in the next day and we changed it. Two patients (8\%) had small superficial pressure sores one on the elbow and one on the scapula which healed by dressing. Figure 3 \& Figure 4 show two of our patients and demonstrates shoulder improvement postoperatively.

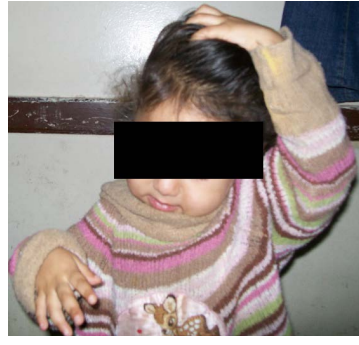

(a)

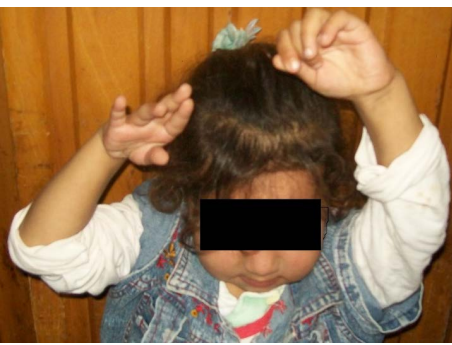

(c)

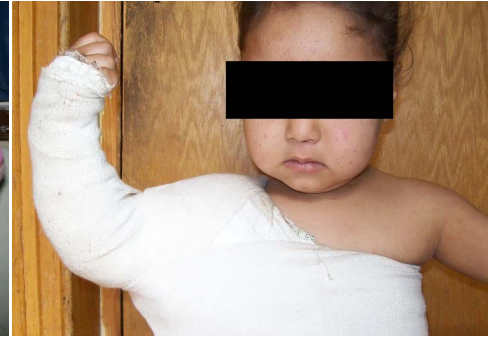

(b)

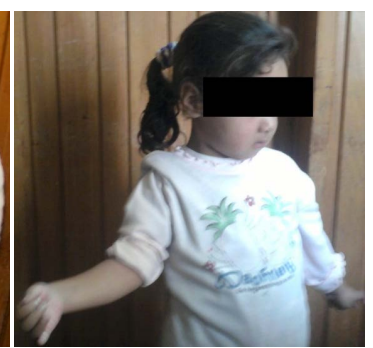

(d)

Figure 3. (a) Preoperative; (b) Before cast removal; (c) Six months postoperative; (d) Twelve months postoperative.

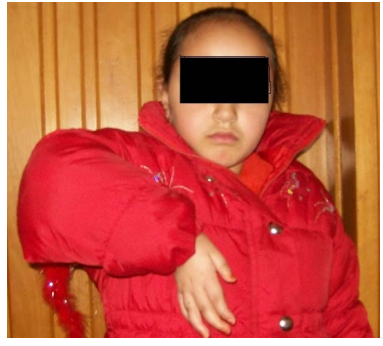

(a)

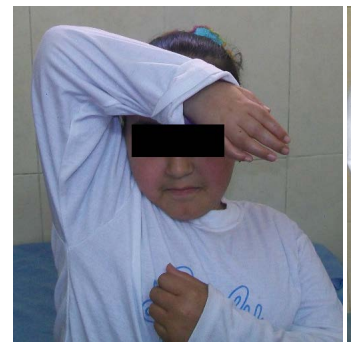

(c)

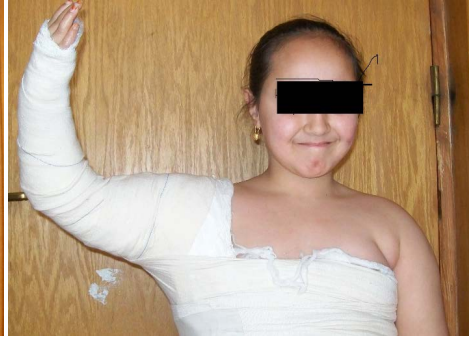

(b)

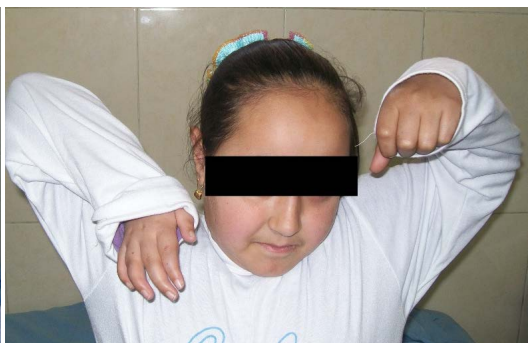

(d)

Figure 4. (a) Preoperative; (b) Before cast removal; (c) \& (d) Six months postoperative. 


\section{Discussion}

Generally, brachial plexus palsy due to obstetric trauma affects about $0.1 \%$ to $0.4 \%$ of births. Surprisingly, about $50 \%$ of these children show spontaneously accepted outcome. However, other children will experience significant shoulder function impairment due to both external rotation and abduction deficits. Additionally, such failure of muscle recovery may cause deformity of the glenohumeral joint. The traumatic process during labour itself may result in myostatic contracture [13].

Some limitation with glenohumeral movement as well as increased scapular winging will be noticed in such cases even if the child has a mild form of chronic brachial palsy. Only infants who will have their antigravity biceps recovered at first 1.5 months of life will not experience shoulder girdle asymmetry on the long-term follow up [14].

The effect of surgery on the glenohumeral joint impairment is still considered a matter of debate. It has been reported that there is a satisfactory improvement of the shoulder mobility as well as correction of the osseocartilaginous deformity [15] [16]. On the other hand, others found no such effects on performing post-operative MR scans [17].

Adducted internally rotated shoulder and glenohumeral deformities were our main concern in this study. In our experience, transfer of latissimus dorsi and teres major muscles accompanied with subscapularis release improved shoulder active motion and decrease passive resistance.

The average age of our patients at the time of surgery was 40.6 months (range 2 - 12 years). This was slightly more as compared to that quoted in western literature. In a series by Waters et al., the average age at the time of tendon transfers was 27 months [18] while that in a series by Hoffer et al., it was 28 months [19]. On the other hand, our results are much nearer to this study coming from Bombay Hospital \& Medical Research Centre in India at which the average age at the time of surgery was 44 months (range 18 months to 7 years) and this mostly due to the similar cultural circumstances [20].

In our study, we included 11 boys (44\%) and 14 girls (56\%). Moreover, the right upper limb was the commonest to be affected when compared to the left one (22 vs. 3 cases).

Another study handling the same perspective included 32 cases; of whom 18 (56.25\%) were boys, and 14 (43.75\%) were girls. Additionally, the right shoulder was affected in 15 cases (46.87\%) while the remaining 17 cases $(53.13 \%)$ had their left shoulder affected [21].

Cohen et al. found very clear functional and clinical improvement provided by subscapularis muscle release with latissimus dorsi and teres major transfer [21]. These previous results are similar to our results which revealed highly favorable outcome after subscapularis release combined with tendons transfer. More than $70 \%$ of patients included in our study reached G IV and G V shoulder function according to Modified Gilbert shoulder evaluation scale. 
An Indian study was conducted to evaluate the clinical outcome of shoulder muscle transfer for shoulder abnormalities in obstetric brachial plexus palsy. Using modified Mallet score, both shoulder movement and functions were significantly improved after the surgical procedure after a mean follow up of 4 years (range 2.5 - 8 years). The pre-operative Mallet score ranged from 8 to 13 with a mean of $11.2 \pm 1.41$, and that score has improved to a mean of $19.2 \pm 1.66$ (range 15 - 20). Moreover, no patient had worsening of shoulder function [20].

Another Egyptian study that was conducted to compare the outcome of subscapularis sliding combined with either latissimus dorsi transfer (group A) or combined latissimus dorsi and teres major transfer (group B) in cases of obstetric brachial palsies. Like this study, they used the modified Gilbert shoulder grading system for assessment. All patients showed statistically significant improvement of active shoulder abduction and external rotation without significant differences between the two groups. On the other hand, Significant external rotation contracture of the shoulder was experienced in $66.67 \%$ of cases but it was more noticed in group B cases. It was concluded that corrective surgery should be restricted to latissimus dorsi transfer without teres major transfer to avoid external rotation contractures [22].

Although many surgeons have advocated the satisfactory results after managing such patients with subscapularis release, latissimus dorsi and teres major tendon transfer, others have noticed a decrease in the post-operative gain on 10-year follow up. However, the end points reached were still higher than untreated patients [23].

A recent study has published that the short-term results of latissimus dorsi transfer and subscapularis release are encouraging. However, these advantages deteriorated over the long follow-up period. Abduction was maintained over the long-term, but external rotation deteriorated. Internal rotation deteriorated over a long-term longitudinal follow-up, leading to functional impairment [24].

Our study has some limitations; it is retrospective single center study that may induce a referral bias. Moreover, the number of included cases was relatively small. Additionally, this study lacks the radiological follow up during the post-operative period and that is because it is our center policy to depend on clinical examination for postoperative evaluation. The short follow up periods can't make us form a solid consensus about this deterioration of shoulder function after long time of surgery.

\section{Conclusion}

Tendon transfer is a valid easy procedure for correction of shoulder deformities in patients with obstetrical brachial plexus palsy. It is considered a very good option for patients who missed the chance of microsurgical repair or patients with poor shoulder recovery after surgery. Although some authors reported deterioration of shoulder function with log time follow up after tendon transfer it is still better than those who were not operated. 


\section{Conflicts of Interest}

The author declares no conflicts of interest regarding the publication of this paper.

\section{References}

[1] Yang, L.J.-S., McGillicuddy, J.E. and Chimbira, W. (2011) Clinical Presentation and Considerations of Neonatal Brachial Plexus Palsy. In: Practical Management of Pediatric and Adult Brachial Plexus Palsies E-Book. Expert Consult. Online, Print, and DVD, Elsevier Inc., Amsterdam, 35.

https://doi.org/10.1016/B978-1-4377-0575-1.00004-6

[2] Evans-Jones, G., Kay, S., Weindling, A., Cranny, G., Ward, A., Bradshaw, A. and Hernon, C. (2003) Congenital Brachial Palsy: Incidence, Causes, and Outcome in the United Kingdom and Republic of Ireland. Archives of Disease in Childhood-Fetal and Neonatal Edition, 88, F185-F189.

https://doi.org/10.1136/fn.88.3.F185

[3] Jacoby, M. (2008) The Birth of Kaiser William II (1859-1941) and His Birth Injury. Journal of Medical Biography, 16, 178-183. https://doi.org/10.1258/jmb.2007.007030

[4] Alanen, M., Halonen, J.-P., Katevuo, K. and Vilkki, P. (1986) Early Surgical Exploration and Epineural Repair in Birth Brachial Palsy. Zeitschrift für Kinderchirurgie, 41, 335-337. https://doi.org/10.1055/s-2008-1043371

[5] Gordon, M., Rich, H., Deutschberger, J. and Green, M. (1973) The Immediate and Long-Term Outcome of Obstetric Birth Trauma: I. Brachial Plexus Paralysis. American Journal of Obstetrics and Gynecology, 117, 51-56. https://doi.org/10.1016/0002-9378(73)90727-8

[6] Bennet, G.C. and Harrold, A. (1976) Prognosis and Early Management of Birth Injuries to the Brachial Plexus. British Medical Journal, 1, 1520-1521. https://doi.org/10.1136/bmj.1.6024.1520

[7] Hoeksma, A.F., Ter Steeg, A.M., Nelissen, R.G., Van Ouwerkerk, W.J., Lankhorst, G.J. and De Jong, B.A. (2004) Neurological Recovery in Obstetric Brachial Plexus Injuries: An Historical Cohort Study. Developmental Medicine and Child Neurology, 46, 76-83. https://doi.org/10.1017/S0012162204000179

[8] Hoeksma, A.F., Ter Steeg, A.M., Dijkstra, P., Nelissen, R.G., Beelen, A. and De Jong, B.A. (2003) Shoulder Contracture and Osseous Deformity in Obstetrical Brachial Plexus Injuries. JBJS, 85, 316-322. https://doi.org/10.2106/00004623-200302000-00020

[9] Nath, R. and Paizi, M. (2007) Improvement in Abduction of the Shoulder after Reconstructive Soft-Tissue Procedures in Obstetric Brachial Plexus Palsy. The Journal of Bone and Joint Surgery. British Volume, 89, 620-626. https://doi.org/10.1302/0301-620X.89B5.18403

[10] Terzis, J.K. and Kokkalis, Z.T. (2008) Outcomes of Secondary Shoulder Reconstruction in Obstetrical Brachial Plexus Palsy. Plastic and Reconstructive Surgery, 122, 1812-1822. https://doi.org/10.1097/01.prs.0000305537.74910.bf

[11] Newman, C.J., Morrison, L., Lynch, B. and Hynes, D. (2006) Outcome of Subscapularis Muscle Release for Shoulder Contracture Secondary to Brachial Plexus Palsy at Birth. Journal of Pediatric Orthopaedics, 26, 647-651. https://doi.org/10.1097/01.bpo.0000233806.72423.30

[12] Grossman, J., Price, A., Tidwell, M., Ramos, L., Alfonso, I. and Yaylali, I. (2003) 
Outcome after Later Combined Brachial Plexus and Shoulder Surgery after Birth Trauma. The Journal of Bone and Joint Surgery. British Volume, 85, 1166-1168. https://doi.org/10.1302/0301-620X.86B6.0860933a

[13] Pöyhiä, T.H., Koivikko, M.P., Peltonen, J.I., Kirjavainen, M.O., Lamminen, A.E. and Nietosvaara, A.Y. (2007) Muscle Changes in Brachial Plexus Birth Injury with Elbow Flexion Contracture: an MRI Study. Pediatric Radiology, 37, 173-179. https://doi.org/10.1007/s00247-006-0374-0

[14] Pearl, M.L. (2009) Shoulder Problems in Children with Brachial Plexus Birth Palsy: Evaluation and Management. JAAOS Journal of the American Academy of Orthopaedic Surgeons, 17, 242-254. https://doi.org/10.5435/00124635-200904000-00005

[15] El-Gammal, T.A., Saleh, W.R., El-Sayed, A., Kotb, M.M., Imam, H.M. and Fathi, N.A. (2006) Tendon Transfer around the Shoulder in Obstetric Brachial Plexus Paralysis: Clinical and Computed Tomographic Study. Journal of Pediatric Orthopaedics, 26, 641-646. https://doi.org/10.1097/01.bpo.0000229975.86188.c4

[16] Hui, J.H. and Torode, I.P. (2003) Changing Glenoid Version after Open Reduction of Shoulders in Children with Obstetric Brachial Plexus Palsy. Journal of Pediatric Orthopaedics, 23, 109-113. https://doi.org/10.1097/01241398-200301000-00022

[17] Kozin, S.H., Chafetz, R.S., Barus, D. and Filipone, L. (2006) Magnetic Resonance Imaging and Clinical Findings before and after Tendon Transfers about the Shoulder in Children with Residual Brachial Plexus Birth Palsy. Journal of Shoulder and Elbow Surgery, 15, 554-561. https://doi.org/10.1097/BPO.0b013e3181cfce09

[18] Waters, P.M. (2010) Management of Shoulder Deformities in Brachial Plexus Birth Palsies. Journal of Pediatric Orthopaedics, 30, S53-S56. https://doi.org/10.1097/BPO.0b013e3181c1893d

[19] Hoffer, M.M. and Phipps, G.J. (1998) Closed Reduction and Tendon Transfer for Treatment of Dislocation of the Glenohumeral Joint Secondary to Brachial Plexus Birth Palsy. JBJS, 80, 997-1001. https://doi.org/10.2106/00004623-199807000-00008

[20] Thatte, M.R., Agashe, M.V., Rao, A., Rathod, C.M. and Mehta, R. (2011) Clinical Outcome of Shoulder Muscle Transfer for Shoulder Deformities in Obstetric Brachial Plexus Palsy: A Study of 150 Cases. Indian Journal of Plastic Surgery: Official Publication of the Association of Plastic Surgeons of India, 44, 21. https://doi.org/10.4103/0970-0358.81441

[21] Cohen, G., Rampal, V., Aubart-Cohen, F., Seringe, R. and Wicart, P. (2010) Brachial Plexus Birth Palsy Shoulder Deformity Treatment Using Subscapularis Release Combined to Tendons Transfer. Orthopaedics \& Traumatology. Surgery \& Research, 96, 334-339. https://doi.org/10.1016/j.otsr.2010.02.004

[22] Abdel-Ghani, H., Hamdy, K., Basha, N. and Tarraf, Y. (2012) Tendon Transfer for Treatment of Internal Rotation Contracture of the Shoulder in Brachial Plexus Birth Palsy. Journal of Hand Surgery (European Volume), 37, 781-786.

https://doi.org/10.1177/1753193412451401

[23] Guermazi, M., Ghroubi, S., Mezghanni, M., Triki, F. and Elleuch, M. (2004) Suivi à long terme des épaules paralytiques obstétricales (à propos de 129 cas). Annales de réadaptation et de médecine physique, 47, 7-12.

https://doi.org/10.1016/j.annrmp.2003.06.002

[24] Werthel, J.-D., Wagner, E.R. and Elhassan, B.T. (2018) Long-Term Results of Latissimus Dorsi Transfer for Internal Rotation Contracture of the Shoulder in Patients with Obstetric Brachial Plexus Injury. JSES Open Access, 2, 159-164. https://doi.org/10.1016/j.jses.2018.05.002 